\title{
PROSPECT THEORY: AN ANALYSIS OF DECISION UNDER RISK
}

\section{By DANiEl Kahneman AND Amos TVERsky ${ }^{1}$}

This paper presents a critique of expected utility theory as a descriptive model of decision making under risk, and develops an alternative model, called prospect theory. Choices among risky prospects exhibit several pervasive effects that are inconsistent with the basic tenets of utility theory. In particular, people underweight outcomes that are merely probable in comparison with outcomes that are obtained with certainty. This tendency, called the certainty effect, contributes to risk aversion in choices involving sure gains and to risk seeking in choices involving sure losses. In addition, people generally discard components that are shared by all prospects under consideration. This tendency, called the isolation effect, leads to inconsistent preferences when the same choice is presented in different forms. An alternative theory of choice is developed, in which value is assigned to gains and losses rather than to final assets and in which probabilities are replaced by decision weights. The value function is normally concave for gains, commonly convex for losses, and is generally steeper for losses than for gains. Decision weights are generally lower than the corresponding probabilities, except in the range of low probabilities. Overweighting of low probabilities may contribute to the attractiveness of both insurance and gambling.

\section{INTRODUCTION}

EXPECTED UTILITY THEORY has dominated the analysis of decision making under risk. It has been generally accepted as a normative model of rational choice [24], and widely applied as a descriptive model of economic behavior, e.g. $[\mathbf{1 5}, \mathbf{4}]$. Thus, it is assumed that all reasonable people would wish to obey the axioms of the theory $[\mathbf{4 7}, \mathbf{3 6}]$, and that most people actually do, most of the time.

The present paper describes several classes of choice problems in which preferences systematically violate the axioms of expected utility theory. In the light of these observations we argue that utility theory, as it is commonly interpreted and applied, is not an adequate descriptive model and we propose an alternative account of choice under risk.

\section{CRITIQUE}

Decision making under risk can be viewed as a choice between prospects or gambles. A prospect $\left(x_{1}, p_{1} ; \ldots ; x_{n}, p_{n}\right)$ is a contract that yields outcome $x_{i}$ with probability $p_{i}$, where $p_{1}+p_{2}+\ldots+p_{n}=1$. To simplify notation, we omit null outcomes and use $(x, p)$ to denote the prospect $(x, p ; 0,1-p)$ that yields $x$ with probability $p$ and 0 with probability $1-p$. The (riskless) prospect that yields $x$ with certainty is denoted by $(x)$. The present discussion is restricted to prospects with so-called objective or standard probabilities.

The application of expected utility theory to choices between prospects is based on the following three tenets.

(i) Expectation: $U\left(x_{1}, p_{1} ; \ldots ; x_{n}, p_{n}\right)=p_{1} u\left(x_{1}\right)+\ldots+p_{n} u\left(x_{n}\right)$.

${ }^{1}$ This work was supported in part by grants from the Harry F. Guggenheim Foundation and from the Advanced Research Projects Agency of the Department of Defense and was monitored by Office of Naval Research under Contract N00014-78-C-0100 (ARPA Order No. 3469) under Subcontract 78-072-0722 from Decisions and Designs, Inc. to Perceptronics, Inc. We also thank the Center for Advanced Study in the Behavioral Sciences at Stanford for its support. 
That is, the overall utility of a prospect, denoted by $U$, is the expected utility of its outcomes.

(ii) Asset Integration: $\left(x_{1}, p_{1} ; \ldots ; x_{n}, p_{n}\right)$ is acceptable at asset position $w$ iff $U\left(w+x_{1}, p_{1} ; \ldots ; w+x_{n}, p_{n}\right)>u(w)$.

That is, a prospect is acceptable if the utility resulting from integrating the prospect with one's assets exceeds the utility of those assets alone. Thus, the domain of the utility function is final states (which include one's asset position) rather than gains or losses.

Although the domain of the utility function is not limited to any particular class of consequences, most applications of the theory have been concerned with monetary outcomes. Furthermore, most economic applications introduce the following additional assumption.

(iii) Risk Aversion: $u$ is concave $\left(u^{\prime \prime}<0\right)$.

A person is risk averse if he prefers the certain prospect $(x)$ to any risky prospect with expected value $x$. In expected utility theory, risk aversion is equivalent to the concavity of the utility function. The prevalence of risk aversion is perhaps the best known generalization regarding risky choices. It led the early decision theorists of the eighteenth century to propose that utility is a concave function of money, and this idea has been retained in modern treatments (Pratt [33], Arrow [4]).

In the following sections we demonstrate several phenomena which violate these tenets of expected utility theory. The demonstrations are based on the responses of students and university faculty to hypothetical choice problems. The respondents were presented with problems of the type illustrated below.

Which of the following would you prefer?
A: $50 \%$ chance to win 1,000 ,
B: 450 for sure.
$50 \%$ chance to win nothing;

The outcomes refer to Israeli currency. To appreciate the significance of the amounts involved, note that the median net monthly income for a family is about 3,000 Israeli pounds. The respondents were asked to imagine that they were actually faced with the choice described in the problem, and to indicate the decision they would have made in such a case. The responses were anonymous, and the instructions specified that there was no 'correct' answer to such problems, and that the aim of the study was to find out how people choose among risky prospects. The problems were presented in questionnaire form, with at most a dozen problems per booklet. Several forms of each questionnaire were constructed so that subjects were exposed to the problems in different orders. In addition, two versions of each problem were used in which the left-right position of the prospects was reversed.

The problems described in this paper are selected illustrations of a series of effects. Every effect has been observed in several problems with different outcomes and probabilities. Some of the problems have also been presented to groups of students and faculty at the University of Stockholm and at the 
University of Michigan. The pattern of results was essentially identical to the results obtained from Israeli subjects.

The reliance on hypothetical choices raises obvious questions regarding the validity of the method and the generalizability of the results. We are keenly aware of these problems. However, all other methods that have been used to test utility theory also suffer from severe drawbacks. Real choices can be investigated either in the field, by naturalistic or statistical observations of economic behavior, or in the laboratory. Field studies can only provide for rather crude tests of qualitative predictions, because probabilities and utilities cannot be adequately measured in such contexts. Laboratory experiments have been designed to obtain precise measures of utility and probability from actual choices, but these experimental studies typically involve contrived gambles for small stakes, and a large number of repetitions of very similar problems. These features of laboratory gambling complicate the interpretation of the results and restrict their generality.

By default, the method of hypothetical choices emerges as the simplest procedure by which a large number of theoretical questions can be investigated. The use of the method relies on the assumption that people often know how they would behave in actual situations of choice, and on the further assumption that the subjects have no special reason to disguise their true preferences. If people are reasonably accurate in predicting their choices, the presence of common and systematic violations of expected utility theory in hypothetical problems provides presumptive evidence against that theory.

\section{Certainty, Probability, and Possibility}

In expected utility theory, the utilities of outcomes are weighted by their probabilities. The present section describes a series of choice problems in which people's preferences systematically violate this principle. We first show that people overweight outcomes that are considered certain, relative to outcomes which are merely probable-a phenomenon which we label the certainty effect.

The best known counter-example to expected utility theory which exploits the certainty effect was introduced by the French economist Maurice Allais in 1953 [2]. Allais' example has been discussed from both normative and descriptive standpoints by many authors $[\mathbf{2 8 ,}, \mathbf{3 8}]$. The following pair of choice problems is a variation of Allais' example, which differs from the original in that it refers to moderate rather than to extremely large gains. The number of respondents who answered each problem is denoted by $N$, and the percentage who choose each option is given in brackets.

Problem 1: Choose between
A: 2,500 with probability .33 ,
2,400 with probability .66 ,
0 with probability
.01
$N=72$
[82]* 
Problem 2: Choose between

$$
\begin{aligned}
& \text { C: } 2,500 \text { with probability } .33, \quad \text { D: } 2,400 \text { with probability } .34 \text {, } \\
& 0 \text { with probability } \quad .67 ; \quad 0 \text { with probability } \quad .66 \text {. } \\
& N=72 \quad[83]^{*}
\end{aligned}
$$

The data show that 82 per cent of the subjects chose $B$ in Problem 1 , and 83 per cent of the subjects chose $C$ in Problem 2. Each of these preferences is significant at the .01 level, as denoted by the asterisk. Moreover, the analysis of individual patterns of choice indicates that a majority of respondents (61 per cent) made the modal choice in both problems. This pattern of preferences violates expected utility theory in the manner originally described by Allais. According to that theory, with $u(0)=0$, the first preference implies

$$
u(2,400)>.33 u(2,500)+.66 u(2,400) \text { or } .34 u(2,400)>.33 u(2,500)
$$

while the second preference implies the reverse inequality. Note that Problem 2 is obtained from Problem 1 by eliminating a .66 chance of winning 2400 from both prospects under consideration. Evidently, this change produces a greater reduction in desirability when it alters the character of the prospect from a sure gain to a probable one, than when both the original and the reduced prospects are uncertain.

A simpler demonstration of the same phenomenon, involving only twooutcome gambles is given below. This example is also based on Allais [2].

Problem 3:
A: $(4,000, .80), \quad$ or
B: $(3,000)$.
$N=95 \quad[20]$
$[80]^{*}$

Problem 4:

$$
\begin{aligned}
& \text { C: }(4,000, .20), \quad \text { or } \quad \text { D: }(3,000, .25) \text {. } \\
& N=95 \quad[65]^{*}
\end{aligned}
$$

In this pair of problems as well as in all other problem-pairs in this section, over half the respondents violated expected utility theory. To show that the modal pattern of preferences in Problems 3 and 4 is not compatible with the theory, set $u(0)=0$, and recall that the choice of $B$ implies $u(3,000) / u(4,000)>4 / 5$, whereas the choice of $C$ implies the reverse inequality. Note that the prospect $C=(4,000, .20)$ can be expressed as $(A, .25)$, while the prospect $D=(3,000, .25)$ can be rewritten as $(B, .25)$. The substitution axiom of utility theory asserts that if $B$ is preferred to $A$, then any (probability) mixture $(B, p)$ must be preferred to the mixture $(A, p)$. Our subjects did not obey this axiom. Apparently, reducing the probability of winning from 1.0 to .25 has a greater effect than the reduction from 
.8 to .2 . The following pair of choice problems illustrates the certainty effect with non-monetary outcomes.

\section{Problem 5:}

A: $50 \%$ chance to win a threeweek tour of England, France, and Italy;

$N=72$
B: A one-week tour of England, with certainty.

$[78]^{*}$

\section{Problem 6:}

C: $5 \%$ chance to win a threeweek tour of England, France, and Italy;

$$
N=72 \quad[67]^{*}
$$

D: $10 \%$ chance to win a oneweek tour of England.

The certainty effect is not the only type of violation of the substitution axiom. Another situation in which this axiom fails is illustrated by the following problems.

\section{Problem 7:}
A: $(6,000, .45)$,
B: $(3,000, .90)$.
$N=66 \quad[14]$
$[86]^{*}$

\section{Problem 8:}

$$
\begin{aligned}
& \text { C: }(6,000, .001), \quad D:(3,000, .002) \text {. } \\
& N=66[73]^{*}
\end{aligned}
$$

Note that in Problem 7 the probabilities of winning are substantial (.90 and .45), and most people choose the prospect where winning is more probable. In Problem 8 , there is a possibility of winning, although the probabilities of winning are minuscule $(.002$ and .001$)$ in both prospects. In this situation where winning is possible but not probable, most people choose the prospect that offers the larger gain. Similar results have been reported by MacCrimmon and Larsson [28].

The above problems illustrate common attitudes toward risk or chance that cannot be captured by the expected utility model. The results suggest the following empirical generalization concerning the manner in which the substitution axiom is violated. If $(y, p q)$ is equivalent to $(x, p)$, then $(y, p q r)$ is preferred to $(x, p r), 0<p, q, r<1$. This property is incorporated into an alternative theory, developed in the second part of the paper. 


\section{The Reflection Effect}

The previous section discussed preferences between positive prospects, i.e., prospects that involve no losses. What happens when the signs of the outcomes are reversed so that gains are replaced by losses? The left-hand column of Table I displays four of the choice problems that were discussed in the previous section, and the right-hand column displays choice problems in which the signs of the outcomes are reversed. We use $-x$ to denote the loss of $x$, and $>$ to denote the prevalent preference, i.e., the choice made by the majority of subjects.

TABLE I

Preferences Between Positive and Negative Prospects

\begin{tabular}{|c|c|c|c|c|c|}
\hline \multicolumn{3}{|c|}{ Positive prospects } & \multicolumn{3}{|c|}{ Negative prospects } \\
\hline $\begin{array}{c}\text { Problem 3: } \\
\quad N=95\end{array}$ & $\begin{array}{c}(4,000, .80) \\
{[20]}\end{array}$ & $\begin{array}{c}\quad(3,000) \\
{[80]^{*}}\end{array}$ & $\begin{array}{c}\text { Problem 3': } \\
\qquad N=95\end{array}$ & $\begin{array}{c}(-4,000, .80) \\
{[92]^{*}}\end{array}$ & $\begin{array}{c}>\quad-3,000) . \\
{[8]}\end{array}$ \\
\hline $\begin{array}{l}\text { Problem 4: } \\
\quad N=95\end{array}$ & $\begin{array}{c}(4,000, .20) \\
{[65]^{*}}\end{array}$ & $\begin{array}{c}>(3,000, .25) \\
{[35]}\end{array}$ & $\begin{array}{l}\text { Problem 4': } \\
\qquad N=95\end{array}$ & $\begin{array}{c}(-4,000, .20) \\
{[42]}\end{array}$ & $<(-3,000, .25)$ \\
\hline $\begin{array}{l}\text { Problem 7: } \\
\qquad N=66\end{array}$ & $\begin{array}{c}(3,000, .90) \\
{[86]^{*}}\end{array}$ & $\begin{array}{c}>(6,000, .45) \\
{[14]}\end{array}$ & $\begin{array}{l}\text { Problem 7': } \\
\qquad N=66\end{array}$ & $\begin{array}{c}(-3,000, .90) \\
{[8]}\end{array}$ & $\begin{array}{c}<(-6,000, .45) \\
{[92]^{*}}\end{array}$ \\
\hline $\begin{array}{l}\text { Problem 8: } \\
\quad N=66\end{array}$ & $\begin{array}{c}(3,000, .002) \\
{[27]}\end{array}$ & $\begin{array}{c}<\frac{(6,000, .001)}{[73]^{*}} . \\
.\end{array}$ & $\begin{array}{l}\text { Problem 8': } \\
\qquad N=66\end{array}$ & $\begin{array}{c}(-3,000, .002) \\
{[70]^{*}}\end{array}$ & $\begin{array}{c}>(-6,000, .001) \\
{[30]}\end{array}$ \\
\hline
\end{tabular}

In each of the four problems in Table I the preference between negative prospects is the mirror image of the preference between positive prospects. Thus, the reflection of prospects around 0 reverses the preference order. We label this pattern the reflection effect.

Let us turn now to the implications of these data. First, note that the reflection effect implies that risk aversion in the positive domain is accompanied by risk seeking in the negative domain. In Problem 3', for example, the majority of subjects were willing to accept a risk of .80 to lose 4,000 , in preference to a sure loss of 3,000, although the gamble has a lower expected value. The occurrence of risk seeking in choices between negative prospects was noted early by Markowitz [29]. Williams [48] reported data where a translation of outcomes produces a dramatic shift from risk aversion to risk seeking. For example, his subjects were indifferent between $(100, .65 ;-100, .35)$ and $(0)$, indicating risk aversion. They were also indifferent between $(-200, .80)$ and $(-100)$, indicating risk seeking. A recent review by Fishburn and Kochenberger [14] documents the prevalence of risk seeking in choices between negative prospects.

Second, recall that the preferences between the positive prospects in Table I are inconsistent with expected utility theory. The preferences between the corresponding negative prospects also violate the expectation principle in the same manner. For example, Problems $3^{\prime}$ and $4^{\prime}$, like Problems 3 and 4, demonstrate that outcomes which are obtained with certainty are overweighted relative to uncertain outcomes. In the positive domain, the certainty effect contributes to a risk averse preference for a sure gain over a larger gain that is merely probable. In the negative domain, the same effect leads to a risk seeking preference for a loss 
that is merely probable over a smaller loss that is certain. The same psychological principle - the overweighting of certainty-favors risk aversion in the domain of gains and risk seeking in the domain of losses.

Third, the reflection effect eliminates aversion for uncertainty or variability as an explanation of the certainty effect. Consider, for example, the prevalent preferences for $(3,000)$ over $(4,000, .80)$ and for $(4,000, .20)$ over $(3,000, .25)$. To resolve this apparent inconsistency one could invoke the assumption that people prefer prospects that have high expected value and small variance (see, e.g., Allais [2]; Markowitz [30]; Tobin [41]). Since $(3,000)$ has no variance while $(4,000, .80)$ has large variance, the former prospect could be chosen despite its lower expected value. When the prospects are reduced, however, the difference in variance between $(3,000, .25)$ and $(4,000, .20)$ may be insufficient to overcome the difference in expected value. Because $(-3,000)$ has both higher expected value and lower variance than $(-4,000, .80)$, this account entails that the sure loss should be preferred, contrary to the data. Thus, our data are incompatible with the notion that certainty is generally desirable. Rather, it appears that certainty increases the aversiveness of losses as well as the desirability of gains.

\section{Probabilistic Insurance}

The prevalence of the purchase of insurance against both large and small losses has been regarded by many as strong evidence for the concavity of the utility function for money. Why otherwise would people spend so much money to purchase insurance policies at a price that exceeds the expected actuarial cost? However, an examination of the relative attractiveness of various forms of insurance does not support the notion that the utility function for money is concave everywhere. For example, people often prefer insurance programs that offer limited coverage with low or zero deductible over comparable policies that offer higher maximal coverage with higher deductibles-contrary to risk aversion (see, e.g., Fuchs [16]). Another type of insurance problem in which people's responses are inconsistent with the concavity hypothesis may be called probabilistic insurance. To illustrate this concept, consider the following problem, which was presented to 95 Stanford University students.

PROBLEM 9: Suppose you consider the possibility of insuring some property against damage, e.g., fire or theft. After examining the risks and the premium you find that you have no clear preference between the options of purchasing insurance or leaving the property uninsured.

It is then called to your attention that the insurance company offers a new program called probabilistic insurance. In this program you pay half of the regular premium. In case of damage, there is a 50 per cent chance that you pay the other half of the premium and the insurance company covers all the losses; and there is a 50 per cent chance that you get back your insurance payment and suffer all the losses. For example, if an accident occurs on an odd day of the month, you pay the other half of the regular premium and your losses are covered; but if the accident 
occurs on an even day of the month, your insurance payment is refunded and your losses are not covered.

Recall that the premium for full coverage is such that you find this insurance barely worth its cost.

Under these circumstances, would you purchase probabilistic insurance:

$$
N=95 \quad \begin{array}{lll} 
& \text { Yes, No. } \\
N 20] & {[80]^{*}}
\end{array}
$$

Although Problem 9 may appear contrived, it is worth noting that probabilistic insurance represents many forms of protective action where one pays a certain cost to reduce the probability of an undesirable event-without eliminating it altogether. The installation of a burglar alarm, the replacement of old tires, and the decision to stop smoking can all be viewed as probabilistic insurance.

The responses to Problem 9 and to several other variants of the same question indicate that probabilistic insurance is generally unattractive. Apparently, reducing the probability of a loss from $p$ to $p / 2$ is less valuable than reducing the probability of that loss from $p / 2$ to 0 .

In contrast to these data, expected utility theory (with a concave $u$ ) implies that probabilistic insurance is superior to regular insurance. That is, if at asset position $w$ one is just willing to pay a premium $y$ to insure against a probability $p$ of losing $x$, then one should definitely be willing to pay a smaller premium $r y$ to reduce the probability of losing $x$ from $p$ to $(1-r) p, 0<r<1$. Formally, if one is indifferent between $(w-x, p ; w, 1-p)$ and $(w-y)$, then one should prefer probabilistic insurance $(w-x,(1-r) p ; w-y, r p ; w-r y, 1-p)$ over regular insurance $(w-y)$.

To prove this proposition, we show that

$$
p u(w-x)+(1-p) u(w)=u(w-y)
$$

implies

$$
(1-r) p u(w-x)+r p u(w-y)+(1-p) u(w-r y)>u(w-y) .
$$

Without loss of generality, we can set $u(w-x)=0$ and $u(w)=1$. Hence, $u(w-$ $y)=1-p$, and we wish to show that

$$
r p(1-p)+(1-p) u(w-r y)>1-p \quad \text { or } \quad u(w-r y)>1-r p
$$

which holds if and only if $u$ is concave.

This is a rather puzzling consequence of the risk aversion hypothesis of utility theory, because probabilistic insurance appears intuitively riskier than regular insurance, which entirely eliminates the element of risk. Evidently, the intuitive notion of risk is not adequately captured by the assumed concavity of the utility function for wealth.

The aversion for probabilistic insurance is particularly intriguing because all insurance is, in a sense, probabilistic. The most avid buyer of insurance remains vulnerable to many financial and other risks which his policies do not cover. There appears to be a significant difference between probabilistic insurance and what may be called contingent insurance, which provides the certainty of coverage for a 
specified type of risk. Compare, for example, probabilistic insurance against all forms of loss or damage to the contents of your home and contingent insurance that eliminates all risk of loss from theft, say, but does not cover other risks, e.g., fire. We conjecture that contingent insurance will be generally more attractive than probabilistic insurance when the probabilities of unprotected loss are equated. Thus, two prospects that are equivalent in probabilities and outcomes could have different values depending on their formulation. Several demonstrations of this general phenomenon are described in the next section.

\section{The Isolation Effect}

In order to simplify the choice between alternatives, people often disregard components that the alternatives share, and focus on the components that distinguish them (Tversky [44]). This approach to choice problems may produce inconsistent preferences, because a pair of prospects can be decomposed into common and distinctive components in more than one way, and different decompositions sometimes lead to different preferences. We refer to this phenomenon as the isolation effect.

Problem 10: Consider the following two-stage game. In the first stage, there is a probability of .75 to end the game without winning anything, and a probability of .25 to move into the second stage. If you reach the second stage you have a choice between

$$
(4,000, .80) \text { and }(3,000) \text {. }
$$

Your choice must be made before the game starts, i.e., before the outcome of the first stage is known.

Note that in this game, one has a choice between $.25 \times .80=.20$ chance to win 4,000 , and a $.25 \times 1.0=.25$ chance to win 3,000 . Thus, in terms of final outcomes and probabilities one faces a choice between $(4,000, .20)$ and $(3,000, .25)$, as in Problem 4 above. However, the dominant preferences are different in the two problems. Of 141 subjects who answered Problem 10,78 per cent chose the latter prospect, contrary to the modal preference in Problem 4. Evidently, people ignored the first stage of the game, whose outcomes are shared by both prospects, and considered Problem 10 as a choice between $(3,000)$ and $(4,000, .80)$, as in Problem 3 above.

The standard and the sequential formulations of Problem 4 are represented as decision trees in Figures 1 and 2, respectively. Following the usual convention, squares denote decision nodes and circles denote chance nodes. The essential difference between the two representations is in the location of the decision node. In the standard form (Figure 1), the decision maker faces a choice between two risky prospects, whereas in the sequential form (Figure 2) he faces a choice between a risky and a riskless prospect. This is accomplished by introducing a dependency between the prospects without changing either probabilities or 


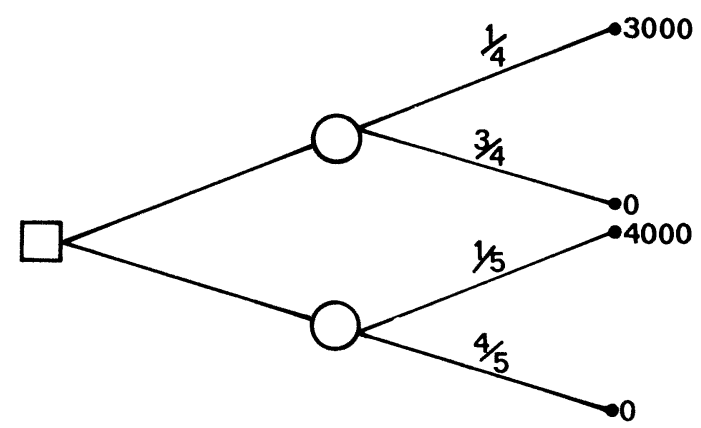

FIGURE 1.-The representation of Problem 4 as a decision tree (standard formulation).

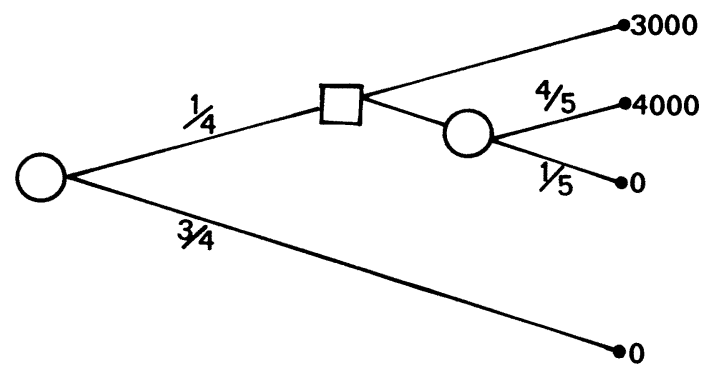

FIGURE 2.-The representation of Problem 10 as a decision tree (sequential formulation).

outcomes. Specifically, the event 'not winning 3,000' is included in the event 'not winning 4,000' in the sequential formulation, while the two events are independent in the standard formulation. Thus, the outcome of winning 3,000 has a certainty advantage in the sequential formulation, which it does not have in the standard formulation.

The reversal of preferences due to the dependency among events is particularly significant because it violates the basic supposition of a decision-theoretical analysis, that choices between prospects are determined solely by the probabilities of final states.

It is easy to think of decision problems that are most naturally represented in one of the forms above rather than in the other. For example, the choice between two different risky ventures is likely to be viewed in the standard form. On the other hand, the following problem is most likely to be represented in the sequential form. One may invest money in a venture with some probability of losing one's capital if the venture fails, and with a choice between a fixed agreed return and a percentage of earnings if it succeeds. The isolation effect implies that the contingent certainty of the fixed return enhances the attractiveness of this option, relative to a risky venture with the same probabilities and outcomes. 
The preceding problem illustrated how preferences may be altered by different representations of probabilities. We now show how choices may be altered by varying the representation of outcomes.

Consider the following problems, which were presented to two different groups of subjects.

Problem 11: In addition to whatever you own, you have been given 1,000. You are now asked to choose between
A: $(1,000, .50), \quad$ and
B: (500).
$N=70 \quad[16]$
$[84]^{*}$

Problem 12: In addition to whatever you own, you have been given 2,000. You are now asked to choose between

$$
\begin{aligned}
& \mathrm{C}:(-1,000, .50), \quad \text { and } \mathrm{D}:(-500) \text {. } \\
& N=68 \quad\left[69^{*}\right]
\end{aligned}
$$

The majority of subjects chose $B$ in the first problem and $C$ in the second. These preferences conform to the reflection effect observed in Table I, which exhibits risk aversion for positive prospects and risk seeking for negative ones. Note, however, that when viewed in terms of final states, the two choice problems are identical. Specifically,

$$
\mathrm{A}=(2,000, .50 ; 1,000, .50)=\mathrm{C}, \quad \text { and } \quad \mathrm{B}=(1,500)=\mathrm{D} \text {. }
$$

In fact, Problem 12 is obtained from Problem 11 by adding 1,000 to the initial bonus, and subtracting 1,000 from all outcomes. Evidently, the subjects did not integrate the bonus with the prospects. The bonus did not enter into the comparison of prospects because it was common to both options in each problem.

The pattern of results observed in Problems 11 and 12 is clearly inconsistent with utility theory. In that theory, for example, the same utility is assigned to a wealth of $\$ 100,000$, regardless of whether it was reached from a prior wealth of $\$ 95,000$ or $\$ 105,000$. Consequently, the choice between a total wealth of $\$ 100,000$ and even chances to own $\$ 95,000$ or $\$ 105,000$ should be independent of whether one currently owns the smaller or the larger of these two amounts. With the added assumption of risk aversion, the theory entails that the certainty of owning $\$ 100,000$ should always be preferred to the gamble. However, the responses to Problem 12 and to several of the previous questions suggest that this pattern will be obtained if the individual owns the smaller amount, but not if he owns the larger amount.

The apparent neglect of a bonus that was common to both options in Problems 11 and 12 implies that the carriers of value or utility are changes of wealth, rather than final asset positions that include current wealth. This conclusion is the cornerstone of an alternative theory of risky choice, which is described in the following sections. 


\section{THEORY}

The preceding discussion reviewed several empirical effects which appear to invalidate expected utility theory as a descriptive model. The remainder of the paper presents an alternative account of individual decision making under risk, called prospect theory. The theory is developed for simple prospects with monetary outcomes and stated probabilities, but it can be extended to more involved choices. Prospect theory distinguishes two phases in the choice process: an early phase of editing and a subsequent phase of evaluation. The editing phase consists of a preliminary analysis of the offered prospects, which often yields a simpler representation of these prospects. In the second phase, the edited prospects are evaluated and the prospect of highest value is chosen. We next outline the editing phase, and develop a formal model of the evaluation phase.

The function of the editing phase is to organize and reformulate the options so as to simplify subsequent evaluation and choice. Editing consists of the application of several operations that transform the outcomes and probabilities associated with the offered prospects. The major operations of the editing phase are described below.

Coding. The evidence discussed in the previous section shows that people normally perceive outcomes as gains and losses, rather than as final states of wealth or welfare. Gains and losses, of course, are defined relative to some neutral reference point. The reference point usually corresponds to the current asset position, in which case gains and losses coincide with the actual amounts that are received or paid. However, the location of the reference point, and the consequent coding of outcomes as gains or losses, can be affected by the formulation of the offered prospects, and by the expectations of the decision maker.

Combination. Prospects can sometimes be simplified by combining the probabilities associated with identical outcomes. For example, the prospect $(200, .25 ; 200, .25)$ will be reduced to $(200, .50)$. and evaluated in this form.

Segregation. Some prospects contain a riskless component that is segregated from the risky component in the editing phase. For example, the prospect $(300, .80 ; 200, .20)$ is naturally decomposed into a sure gain of 200 and the risky prospect $(100, .80)$. Similarly, the prospect $(-400, .40 ;-100, .60)$ is readily seen to consist of a sure loss of 100 and of the prospect $(-300, .40)$.

The preceding operations are applied to each prospect separately. The following operation is applied to a set of two or more prospects.

Cancellation. The essence of the isolation effects described earlier is the discarding of components that are shared by the offered prospects. Thus, our respondents apparently ignored the first stage of the sequential game presented in Problem 10, because this stage was common to both options, and they evaluated the prospects with respect to the results of the second stage (see Figure 2). Similarly, they neglected the common bonus that was added to the prospects in Problems 11 and 12. Another type of cancellation involves the discarding of common constituents, i.e., outcome-probability pairs. For example, the choice 
between $(200, .20 ; 100, .50 ;-50, .30)$ and $(200, .20 ; 150, .50 ;-100, .30)$ can be reduced by cancellation to a choice between $(100, .50 ;-50, .30)$ and $(150, .50 ;-100, .30)$.

Two additional operations that should be mentioned are simplification and the detection of dominance. The first refers to the simplification of prospects by rounding probabilities or outcomes. For example, the prospect $(101, .49)$ is likely to be recoded as an even chance to win 100 . A particularly important form of simplification involves the discarding of extremely unlikely outcomes. The second operation involves the scanning of offered prospects to detect dominated alternatives, which are rejected without further evaluation.

Because the editing operations facilitate the task of decision, it is assumed that they are performed whenever possible. However, some editing operations either permit or prevent the application of others. For example, $(500, .20 ; 101, .49)$ will appear to dominate $(500, .15 ; 99, .51)$ if the second constituents of both prospects are simplified to $(100, .50)$. The final edited prospects could, therefore, depend on the sequence of editing operations, which is likely to vary with the structure of the offered set and with the format of the display. A detailed study of this problem is beyond the scope of the present treatment. In this paper we discuss choice problems where it is reasonable to assume either that the original formulation of the prospects leaves no room for further editing, or that the edited prospects can be specified without ambiguity.

Many anomalies of preference result from the editing of prospects. For example, the inconsistencies associated with the isolation effect result from the cancellation of common components. Some intransitivities of choice are explained by a simplification that eliminates small differences between prospects (see Tversky [43]). More generally, the preference order between prospects need not be invariant across contexts, because the same offered prospect could be edited in different ways depending on the context in which it appears.

Following the editing phase, the decision maker is assumed to evaluate each of the edited prospects, and to choose the prospect of highest value. The overall value of an edited prospect, denoted $V$, is expressed in terms of two scales, $\pi$ and $v$.

The first scale, $\pi$, associates with each probability $p$ a decision weight $\pi(p)$, which reflects the impact of $p$ on the over-all value of the prospect. However, $\pi$ is not a probability measure, and it will be shown later that $\pi(p)+\pi(1-p)$ is typically less than unity. The second scale, $v$, assigns to each outcome $x$ a number $v(x)$, which reflects the subjective value of that outcome. Recall that outcomes are defined relative to a reference point, which serves as the zero point of the value scale. Hence, $v$ measures the value of deviations from that reference point, i.e., gains and losses.

The present formulation is concerned with simple prospects of the form $(x, p ; y, q)$, which have at most two non-zero outcomes. In such a prospect, one receives $x$ with probability $p, y$ with probability $q$, and nothing with probability $1-p-q$, where $p+q \leqslant 1$. An offered prospect is strictly positive if its outcomes are all positive, i.e., if $x, y>0$ and $p+q=1$; it is strictly negative if its outcomes 
are all negative. A prospect is regular if it is neither strictly positive nor strictly negative.

The basic equation of the theory describes the manner in which $\pi$ and $v$ are combined to determine the over-all value of regular prospects.

If $(x, p ; y, q)$ is a regular prospect (i.e., either $p+q<1$, or $x \geqslant 0 \geqslant y$, or $x \leqslant 0 \leqslant$ $y)$, then

$$
V(x, p ; y, q)=\pi(p) v(x)+\pi(q) v(y)
$$

where $v(0)=0, \pi(0)=0$, and $\pi(1)=1$. As in utility theory, $V$ is defined on prospects, while $v$ is defined on outcomes. The two scales coincide for sure prospects, where $V(x, 1.0)=V(x)=v(x)$.

Equation (1) generalizes expected utility theory by relaxing the expectation principle. An axiomatic analysis of this representation is sketched in the Appendix, which describes conditions that ensure the existence of a unique $\pi$ and a ratio-scale $v$ satisfying equation (1).

The evaluation of strictly positive and strictly negative prospects follows a different rule. In the editing phase such prospects are segregated into two components: (i) the riskless component, i.e., the minimum gain or loss which is certain to be obtained or paid; (ii) the risky component, i.e., the additional gain or loss which is actually at stake. The evaluation of such prospects is described in the next equation.

If $p+q=1$ and either $x>y>0$ or $x<y<0$, then

$$
V(x, p ; y, q)=v(y)+\pi(p)[v(x)-v(y)] .
$$

That is, the value of a strictly positive or strictly negative prospect equals the value of the riskless component plus the value-difference between the outcomes, multiplied by the weight associated with the more extreme outcome. For example, $V(400, .25 ; 100, .75)=v(100)+\pi(.25)[v(400)-v(100)]$. The essential feature of equation (2) is that a decision weight is applied to the value-difference $v(x)-v(y)$, which represents the risky component of the prospect, but not to $v(y)$, which represents the riskless component. Note that the right-hand side of equation (2) equals $\pi(p) v(x)+[1-\pi(p)] v(y)$. Hence, equation (2) reduces to equation (1) if $\pi(p)+\pi(1-p)=1$. As will be shown later, this condition is not generally satisfied.

Many elements of the evaluation model have appeared in previous attempts to modify expected utility theory. Markowitz [29] was the first to propose that utility be defined on gains and losses rather than on final asset positions, an assumption which has been implicitly accepted in most experimental measurements of utility (see, e.g., $[7,32])$. Markowitz also noted the presence of risk seeking in preferences among positive as well as among negative prospects, and he proposed a utility function which has convex and concave regions in both the positive and the negative domains. His treatment, however, retains the expectation principle; hence it cannot account for the many violations of this principle; see, e.g., Table I.

The replacement of probabilities by more general weights was proposed by Edwards [9], and this model was investigated in several empirical studies (e.g., 
$[\mathbf{3}, \mathbf{4 2}])$. Similar models were developed by Fellner [12], who introduced the concept of decision weight to explain aversion for ambiguity, and by van Dam [46] who attempted to scale decision weights. For other critical analyses of expected utility theory and alternative choice models, see Allais [2], Coombs [6], Fishburn [13], and Hansson [22].

The equations of prospect theory retain the general bilinear form that underlies expected utility theory. However, in order to accomodate the effects described in the first part of the paper, we are compelled to assume that values are attached to changes rather than to final states, and that decision weights do not coincide with stated probabilities. These departures from expected utility theory must lead to normatively unacceptable consequences, such as inconsistencies, intransitivities, and violations of dominance. Such anomalies of preference are normally corrected by the decision maker when he realizes that his preferences are inconsistent, intransitive, or inadmissible. In many situations, however, the decision maker does not have the opportunity to discover that his preferences could violate decision rules that he wishes to obey. In these circumstances the anomalies implied by prospect theory are expected to occur.

\section{The Value Function}

An essential feature of the present theory is that the carriers of value are changes in wealth or welfare, rather than final states. This assumption is compatible with basic principles of perception and judgment. Our perceptual apparatus is attuned to the evaluation of changes or differences rather than to the evaluation of absolute magnitudes. When we respond to attributes such as brightness, loudness, or temperature, the past and present context of experience defines an adaptation level, or reference point, and stimuli are perceived in relation to this reference point [23]. Thus, an object at a given temperature may be experienced as hot or cold to the touch depending on the temperature to which one has adapted. The same principle applies to non-sensory attributes such as health, prestige, and wealth. The same level of wealth, for example, may imply abject poverty for one person and great riches for another-depending on their current assets.

The emphasis on changes as the carriers of value should not be taken to imply that the value of a particular change is independent of initial position. Strictly speaking, value should be treated as a function in two arguments: the asset position that serves as reference point, and the magnitude of the change (positive or negative) from that reference point. An individual's attitude to money, say, could be described by a book, where each page presents the value function for changes at a particular asset position. Clearly, the value functions described on different pages are not identical: they are likely to become more linear with increases in assets. However, the preference order of prospects is not greatly altered by small or even moderate variations in asset position. The certainty equivalent of the prospect $(1,000, .50)$, for example, lies between 300 and 400 for most people, in a wide range of asset positions. Consequently, the representation 
of value as a function in one argument generally provides a satisfactory approximation.

Many sensory and perceptual dimensions share the property that the psychological response is a concave function of the magnitude of physical change. For example, it is easier to discriminate between a change of $3^{\circ}$ and a change of $6^{\circ}$ in room temperature, than it is to discriminate between a change of $13^{\circ}$ and a change of $16^{\circ}$. We propose that this principle applies in particular to the evaluation of monetary changes. Thus, the difference in value between a gain of 100 and a gain of 200 appears to be greater than the difference between a gain of 1,100 and a gain of 1,200 . Similarly, the difference between a loss of 100 and a loss of 200 appears greater than the difference between a loss of 1,100 and a loss of 1,200 , unless the larger loss is intolerable. Thus, we hypothesize that the value function for changes of wealth is normally concave above the reference point $\left(v^{\prime \prime}(x)<0\right.$, for $\left.x>0\right)$ and often convex below it $\left(v^{\prime \prime}(x)>0\right.$, for $\left.x<0\right)$. That is, the marginal value of both gains and losses generally decreases with their magnitude. Some support for this hypothesis has been reported by Galanter and Pliner [17], who scaled the perceived magnitude of monetary and non-monetary gains and losses.

The above hypothesis regarding the shape of the value function was based on responses to gains and losses in a riskless context. We propose that the value function which is derived from risky choices shares the same characteristics, as illustrated in the following problems.

Problem 13:

$$
\begin{array}{ccc}
(6,000, .25), & \text { or } & (4,000, .25 ; 2,000, .25) \\
N=68 \quad[18] & & {[82]^{*}}
\end{array}
$$

Problem 13':

$$
\begin{aligned}
& (-6,000, .25), \quad \text { or } \quad(-4,000, .25 ;-2,000, .25) \text {. } \\
& N=64 \quad[70]^{*}
\end{aligned}
$$

Applying equation 1 to the modal preference in these problems yields

$$
\begin{aligned}
& \pi(.25) v(6,000)<\pi(.25)[v(4,000)+v(2,000)] \quad \text { and } \\
& \pi(.25) v(-6,000)>\pi(.25)[v(-4,000)+v(-2,000)] .
\end{aligned}
$$

Hence, $v(6,000)<v(4,000)+v(2,000)$ and $v(-6,000)>v(-4,000)+v(-2,000)$. These preferences are in accord with the hypothesis that the value function is concave for gains and convex for losses.

Any discussion of the utility function for money must leave room for the effect of special circumstances on preferences. For example, the utility function of an individual who needs $\$ 60,000$ to purchase a house may reveal an exceptionally steep rise near the critical value. Similarly, an individual's aversion to losses may increase sharply near the loss that would compel him to sell his house and move to 
a less desirable neighborhood. Hence, the derived value (utility) function of an individual does not always reflect "pure" attitudes to money, since it could be affected by additional consequences associated with specific amounts. Such perturbations can readily produce convex regions in the value function for gains and concave regions in the value function for losses. The latter case may be more common since large losses often necessitate changes in life style.

A salient characteristic of attitudes to changes in welfare is that losses loom larger than gains. The aggravation that one experiences in losing a sum of money appears to be greater than the pleasure associated with gaining the same amount [17]. Indeed, most people find symmetric bets of the form $(x, .50 ;-x, .50)$ distinctly unattractive. Moreover, the aversiveness of symmetric fair bets generally increases with the size of the stake. That is, if $x>y \geqslant 0$, then $(y, .50 ;-y, .50)$ is preferred to $(x, .50 ;-x, .50)$. According to equation (1), therefore,

$$
v(y)+v(-y)>v(x)+v(-x) \quad \text { and } \quad v(-y)-v(-x)>v(x)-v(y) .
$$

Setting $y=0$ yields $v(x)<-v(-x)$, and letting $y$ approach $x$ yields $v^{\prime}(x)<$ $v^{\prime}(-x)$, provided $v^{\prime}$, the derivative of $v$, exists. Thus, the value function for losses is steeper than the value function for gains.

In summary, we have proposed that the value function is (i) defined on deviations from the reference point; (ii) generally concave for gains and commonly convex for losses; (iii) steeper for losses than for gains. A value function which satisfies these properties is displayed in Figure 3. Note that the proposed $S$-shaped value function is steepest at the reference point, in marked contrast to the utility function postulated by Markowitz [29] which is relatively shallow in that region.

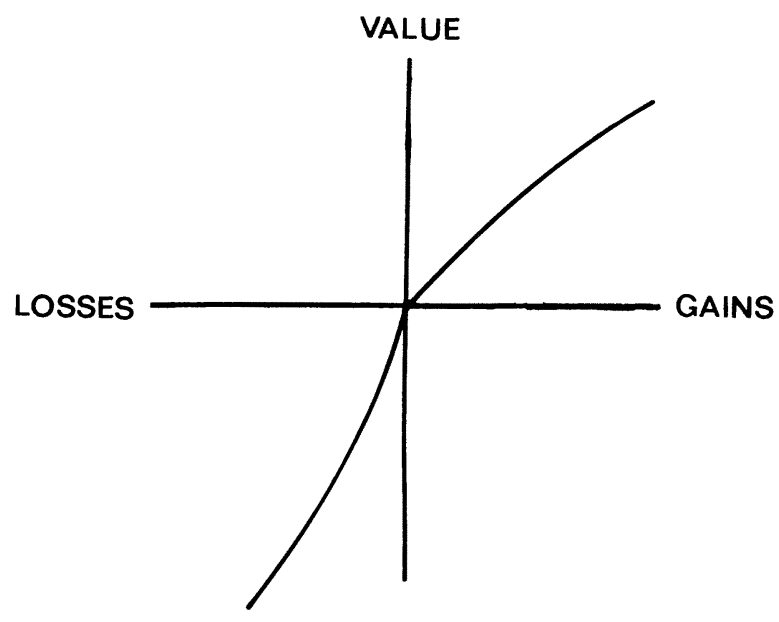

FIGURE 3.-A hypothetical value function. 
Although the present theory can be applied to derive the value function from preferences between prospects, the actual scaling is considerably more complicated than in utility theory, because of the introduction of decision weights. For example, decision weights could produce risk aversion and risk seeking even with a linear value function. Nevertheless, it is of interest that the main properties ascribed to the value function have been observed in a detailed analysis of von Neumann-Morgenstern utility functions for changes of wealth (Fishburn and Kochenberger [14]). The functions had been obtained from thirty decision makers in various fields of business, in five independent studies $[5,18,19,21,40]$. Most utility functions for gains were concave, most functions for losses were convex, and only three individuals exhibited risk aversion for both gains and losses. With a single exception, utility functions were considerably steeper for losses than for gains.

\section{The Weighting Function}

In prospect theory, the value of each outcome is multiplied by a decision weight. Decision weights are inferred from choices between prospects much as subjective probabilities are inferred from preferences in the Ramsey-Savage approach. However, decision weights are not probabilities: they do not obey the probability axioms and they should not be interpreted as measures of degree or belief.

Consider a gamble in which one can win 1,000 or nothing, depending on the toss of a fair coin. For any reasonable person, the probability of winning is .50 in this situation. This can be verified in a variety of ways, e.g., by showing that the subject is indifferent between betting on heads or tails, or by his verbal report that he considers the two events equiprobable. As will be shown below, however, the decision weight $\pi(.50)$ which is derived from choices is likely to be smaller than .50. Decision weights measure the impact of events on the desirability of prospects, and not merely the perceived likelihood of these events. The two scales coincide (i.e., $\pi(p)=p$ ) if the expectation principle holds, but not otherwise.

The choice problems discussed in the present paper were formulated in terms of explicit numerical probabilities, and our analysis assumes that the respondents adopted the stated values of $p$. Furthermore, since the events were identified only by their stated probabilities, it is possible in this context to express decision weights as a function of stated probability. In general, however, the decision weight attached to an event could be influenced by other factors, e.g., ambiguity $[\mathbf{1 0}, \mathbf{1 1}]$.

We turn now to discuss the salient properties of the weighting function $\pi$, which relates decision weights to stated probabilities. Naturally, $\pi$ is an increasing function of $p$, with $\pi(0)=0$ and $\pi(1)=1$. That is, outcomes contingent on an impossible event are ignored, and the scale is normalized so that $\pi(p)$ is the ratio of the weight associated with the probability $p$ to the weight associated with the certain event.

We first discuss some properties of the weighting function for small probabilities. The preferences in Problems 8 and $8^{\prime}$ suggest that for small values of $p, \pi$ 
is a subadditive function of $p$, i.e., $\pi(r p)>r \pi(p)$ for $0<r<1$. Recall that in Problem $8,(6,000, .001)$ is preferred to $(3,000, .002)$. Hence

$$
\frac{\pi(.001)}{\pi(.002)}>\frac{v(3,000)}{v(6,000)}>\frac{1}{2} \quad \text { by the concavity of } v \text {. }
$$

The reflected preferences in Problem $8^{\prime}$ yield the same conclusion. The pattern of preferences in Problems 7 and $7^{\prime}$, however, suggests that subadditivity need not hold for large values of $p$.

Furthermore, we propose that very low probabilities are generally overweighted, that is, $\pi(p)>p$ for small $p$. Consider the following choice problems.

Problem 14:

$$
N=72 \quad[72]^{*}, \quad \text { or } \quad \text { (5). }
$$

Problem 14':

$$
\begin{aligned}
& (-5,000, .001), \quad \text { or } \quad(-5) \text {. } \\
& N=72 \quad[17] \quad[83]^{*}
\end{aligned}
$$

Note that in Problem 14, people prefer what is in effect a lottery ticket over the expected value of that ticket. In Problem 14', on the other hand, they prefer a small loss, which can be viewed as the payment of an insurance premium, over a small probability of a large loss. Similar observations have been reported by Markowitz [29]. In the present theory, the preference for the lottery in Problem 14 implies $\pi(.001) v(5,000)>v(5)$, hence $\pi(.001)>v(5) / v(5,000)>.001$, assuming the value function for gains is concave. The readiness to pay for insurance in Problem 14' implies the same conclusion, assuming the value function for losses is convex.

It is important to distinguish overweighting, which refers to a property of decision weights, from the overestimation that is commonly found in the assessment of the probability of rare events. Note that the issue of overestimation does not arise in the present context, where the subject is assumed to adopt the stated value of $p$. In many real-life situations, overestimation and overweighting may both operate to increase the impact of rare events.

Although $\pi(p)>p$ for low probabilities, there is evidence to suggest that, for all $0<p<1, \pi(p)+\pi(1-p)<1$. We label this property subcertainty. It is readily seen that the typical preferences in any version of Allias' example (see, e.g., Problems 1 and 2) imply subcertainty for the relevant value of $p$. Applying 
equation (1) to the prevalent preferences in Problems 1 and 2 yields, respectively,

$$
\begin{aligned}
& v(2,400)>\pi(.66) v(2,400)+\pi(.33) v(2,500), \quad \text { i.e., } \\
& {[1-\pi(.66] v(2,400)>\pi(.33) v(2,500) \quad \text { and }} \\
& \pi(.33) v(2,500)>\pi(.34) v(2,400) ; \quad \text { hence, } \\
& 1-\pi(.66)>\pi(.34), \quad \text { or } \pi(.66)+\pi(.34)<1 .
\end{aligned}
$$

Applying the same analysis to Allais' original example yields $\pi(.89)+\pi(.11)<1$, and some data reported by MacCrimmon and Larsson [28] imply subcertainty for additional values of $p$.

The slope of $\pi$ in the interval $(0,1)$ can be viewed as a measure of the sensitivity of preferences to changes in probability. Subcertainty entails that $\pi$ is regressive with respect to $p$, i.e., that preferences are generally less sensitive to variations of probability than the expectation principle would dictate. Thus, subcertainty captures an essential element of people's attitudes to uncertain events, namely that the sum of the weights associated with complementary events is typically less than the weight associated with the certain event.

Recall that the violations of the substitution axiom discussed earlier in this paper conform to the following rule: If $(x, p)$ is equivalent to $(y, p q)$ then $(x, p r)$ is not preferred to $(y, p q r), 0<p, q, r \leqslant 1$. By equation (1),

$$
\begin{aligned}
& \pi(p) v(x)=\pi(p q) v(y) \quad \text { implies } \quad \pi(p r) v(x) \leqslant \pi(p q r) v(y) ; \text { hence, } \\
& \frac{\pi(p q)}{\pi(p)} \leqslant \frac{\pi(p q r)}{\pi(p r)}
\end{aligned}
$$

Thus, for a fixed ratio of probabilities, the ratio of the corresponding decision weights is closer to unity when the probabilities are low than when they are high. This property of $\pi$, called subproportionality, imposes considerable constraints on the shape of $\pi$ : it holds if and only if $\log \pi$ is a convex function of $\log p$.

It is of interest to note that subproportionality together with the overweighting of small probabilities imply that $\pi$ is subadditive over that range. Formally, it can be shown that if $\pi(p)>p$ and subproportionality holds, then $\pi(r p)>r \pi(p), 0<$ $r<1$, provided $\pi$ is monotone and continuous over $(0,1)$.

Figure 4 presents a hypothetical weighting function which satisfies overweighting and subadditivity for small values of $p$, as well as subcertainty and subproportionality. These properties entail that $\pi$ is relatively shallow in the open interval and changes abruptly near the end-points where $\pi(0)=0$ and $\pi(1)=1$. The sharp drops or apparent discontinuities of $\pi$ at the endpoints are consistent with the notion that there is a limit to how small a decision weight can be attached to an event, if it is given any weight at all. A similar quantum of doubt could impose an upper limit on any decision weight that is less than unity. This quantal effect may reflect the categorical distinction between certainty and uncertainty. On the other hand, the simplification of prospects in the editing phase can lead the individual to discard events of extremely low probability and to treat events of extremely high probability as if they were certain. Because people are limited in 
their ability to comprehend and evaluate extreme probabilities, highly unlikely events are either ignored or overweighted, and the difference between high probability and certainty is either neglected or exaggerated. Consequently, $\pi$ is not well-behaved near the end-points.

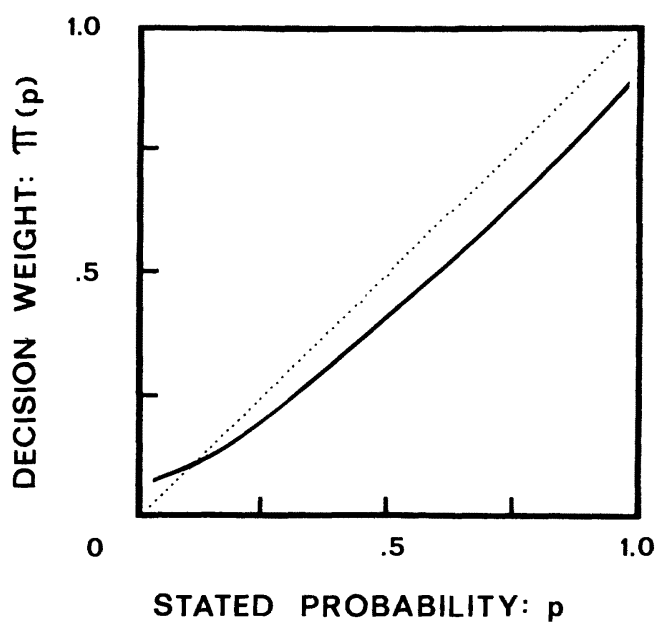

FIGURE 4.-A hypothetical weighting function.

The following example, due to Zeckhauser, illustrates the hypothesized nonlinearity of $\pi$. Suppose you are compelled to play Russian roulette, but are given the opportunity to purchase the removal of one bullet from the loaded gun. Would you pay as much to reduce the number of bullets from four to three as you would to reduce the number of bullets from one to zero? Most people feel that they would be willing to pay much more for a reduction of the probability of death from $1 / 6$ to zero than for a reduction from $4 / 6$ to $3 / 6$. Economic considerations would lead one to pay more in the latter case, where the value of money is presumably reduced by the considerable probability that one will not live to enjoy it.

An obvious objection to the assumption that $\pi(p) \neq p$ involves comparisons between prospects of the form $(x, p ; x, q)$ and $\left(x, p^{\prime} ; x, q^{\prime}\right)$, where $p+q=p^{\prime}+q^{\prime}<$ 1. Since any individual will surely be indifferent between the two prospects, it could be argued that this observation entails $\pi(p)+\pi(q)=\pi\left(p^{\prime}\right)+\pi\left(q^{\prime}\right)$, which in turn implies that $\pi$ is the identity function. This argument is invalid in the present theory, which assumes that the probabilities of identical outcomes are combined in the editing of prospects. A more serious objection to the nonlinearity of $\pi$ involves potential violations of dominance. Suppose $x>y>0, p>p^{\prime}$, and $p+q=$ $p^{\prime}+q^{\prime}<1$; hence, $(x, p ; y, q)$ dominates $\left(x, p^{\prime} ; y, q^{\prime}\right)$. If preference obeys 
dominance, then

$$
\pi(p) v(x)+\pi(q) v(y)>\pi\left(p^{\prime}\right) v(x)+\pi\left(q^{\prime}\right) v(y),
$$

or

$$
\frac{\pi(p)-\pi\left(p^{\prime}\right)}{\pi\left(q^{\prime}\right)-\pi(q)}>\frac{v(y)}{v(x)}
$$

Hence, as $y$ approaches $x, \pi(p)-\pi\left(p^{\prime}\right)$ approaches $\pi\left(q^{\prime}\right)-\pi(q)$. Since $p-p^{\prime}=$ $q^{\prime}-q, \pi$ must be essentially linear, or else dominance must be violated.

Direct violations of dominance are prevented, in the present theory, by the assumption that dominated alternatives are detected and eliminated prior to the evaluation of prospects. However, the theory permits indirect violations of dominance, e.g., triples of prospects so that $A$ is preferred to $B, B$ is preferred to $C$, and $C$ dominates $A$. For an example, see Raiffa [34, p. 75].

Finally, it should be noted that the present treatment concerns the simplest decision task in which a person chooses between two available prospects. We have not treated in detail the more complicated production task (e.g., bidding) where the decision maker generates an alternative that is equal in value to a given prospect. The asymmetry between the two options in this situation could introduce systematic biases. Indeed, Lichtenstein and Slovic [27] have constructed pairs of prospects $A$ and $B$, such that people generally prefer $A$ over $B$, but bid more for $B$ than for $A$. This phenomenon has been confirmed in several studies, with both hypothetical and real gambles, e.g., Grether and Plott [20]. Thus, it cannot be generally assumed that the preference order of prospects can be recovered by a bidding procedure.

Because prospect theory has been proposed as a model of choice, the inconsistency of bids and choices implies that the measurement of values and decision weights should be based on choices between specified prospects rather than on bids or other production tasks. This restriction makes the assessment of $v$ and $\pi$ more difficult because production tasks are more convenient for scaling than pair comparisons.

\section{DISCUSSION}

In the final section we show how prospect theory accounts for observed attitudes toward risk, discuss alternative representations of choice problems induced by shifts of reference point, and sketch several extensions of the present treatment.

\section{Risk Attitudes}

The dominant pattern of preferences observed in Allais' example (Problems 1 and 2) follows from the present theory iff

$$
\frac{\pi(.33)}{\pi(.34)}>\frac{v(2,400)}{v(2,500)}>\frac{\pi(.33)}{1-\pi(.66)}
$$


Hence, the violation of the independence axiom is attributed in this case to subcertainty, and more specifically to the inequality $\pi(.34)<1-\pi(.66)$. This analysis shows that an Allais-type violation will occur whenever the $v$-ratio of the two non-zero outcomes is bounded by the corresponding $\pi$-ratios.

Problems 3 through 8 share the same structure, hence it suffices to consider one pair, say Problems 7 and 8 . The observed choices in these problems are implied by the theory iff

$$
\frac{\pi(.001)}{\pi(.002)}>\frac{v(3,000)}{v(6,000)}>\frac{\pi(.45)}{\pi(.90)}
$$

The violation of the substitution axiom is attributed in this case to the subproportionality of $\pi$. Expected utility theory is violated in the above manner, therefore, whenever the $v$-ratio of the two outcomes is bounded by the respective $\pi$-ratios. The same analysis applies to other violations of the substitution axiom, both in the positive and in the negative domain.

We next prove that the preference for regular insurance over probabilistic insurance, observed in Problem 9, follows from prospect theory-provided the probability of loss is overweighted. That is, if $(-x, p)$ is indifferent to $(-y)$, then $(-y)$ is preferred to $(-x, p / 2 ;-y, p / 2 ;-y / 2,1-p)$. For simplicity, we define for $x \geqslant 0, f(x)=-v(-x)$. Since the value function for losses is convex, $f$ is a concave function of $x$. Applying prospect theory, with the natural extension of equation 2, we wish to show that

$$
\begin{aligned}
& \pi(p) f(x)=f(y) \quad \text { implies } \\
& \begin{aligned}
f(y) & \leqslant f(y / 2)+\pi(p / 2)[f(y)-f(y / 2)]+\pi(p / 2)[f(x)-f(y / 2)] \\
& =\pi(p / 2) f(x)+\pi(p / 2) f(y)+[1-2 \pi(p / 2)] f(y / 2) .
\end{aligned}
\end{aligned}
$$

Substituting for $f(x)$ and using the concavity of $f$, it suffices to show that

$$
f(y) \leqslant \frac{\pi(p / 2)}{\pi(p)} f(y)+\pi(p / 2) f(y)+f(y) / 2-\pi(p / 2) f(y)
$$

or

$$
\pi(p) / 2 \leqslant \pi(p / 2), \quad \text { which follows from the subadditivity of } \pi \text {. }
$$

According to the present theory, attitudes toward risk are determined jointly by $v$ and $\pi$, and not solely by the utility function. It is therefore instructive to examine the conditions under which risk aversion or risk seeking are expected to occur. Consider the choice between the gamble $(x, p)$ and its expected value $(p x)$. If $x>0$, risk seeking is implied whenever $\pi(p)>v(p x) / v(x)$, which is greater than $p$ if the value function for gains is concave. Hence, overweighting $(\pi(p)>p)$ is necessary but not sufficient for risk seeking in the domain of gains. Precisely the same condition is necessary but not sufficient for risk aversion when $x<0$. This analysis restricts risk seeking in the domain of gains and risk aversion in the domain of losses to small probabilities, where overweighting is expected to hold. 
Indeed these are the typical conditions under which lottery tickets and insurance policies are sold. In prospect theory, the overweighting of small probabilities favors both gambling and insurance, while the $S$-shaped value function tends to inhibit both behaviors.

Although prospect theory predicts both insurance and gambling for small probabilities, we feel that the present analysis falls far short of a fully adequate account of these complex phenomena. Indeed, there is evidence from both experimental studies [37], survey research [26], and observations of economic behavior, e.g., service and medical insurance, that the purchase of insurance often extends to the medium range of probabilities, and that small probabilities of disaster are sometimes entirely ignored. Furthermore, the evidence suggests that minor changes in the formulation of the decision problem can have marked effects on the attractiveness of insurance [37]. A comprehensive theory of insurance behavior should consider, in addition to pure attitudes toward uncertainty and money, such factors as the value of security, social norms of prudence, the aversiveness of a large number of small payments spread over time, information and misinformation regarding probabilities and outcomes, and many others. Some effects of these variables could be described within the present framework, e.g., as changes of reference point, transformations of the value function, or manipulations of probabilities or decision weights. Other effects may require the introduction of variables or concepts which have not been considered in this treatment.

\section{Shifts of Reference}

So far in this paper, gains and losses were defined by the amounts of money that are obtained or paid when a prospect is played, and the reference point was taken to be the status quo, or one's current assets. Although this is probably true for most choice problems, there are situations in which gains and losses are coded relative to an expectation or aspiration level that differs from the status quo. For example, an unexpected tax withdrawal from a monthly pay check is experienced as a loss, not as a reduced gain. Similarly, an entrepreneur who is weathering a slump with greater success than his competitors may interpret a small loss as a gain, relative to the larger loss he had reason to expect.

The reference point in the preceding examples corresponded to an asset position that one had expected to attain. A discrepancy between the reference point and the current asset position may also arise because of recent changes in wealth to which one has not yet adapted [29]. Imagine a person who is involved in a business venture, has already lost 2,000 and is now facing a choice between a sure gain of 1,000 and an even chance to win 2,000 or nothing. If he has not yet adapted to his losses, he is likely to code the problem as a choice between $(-2,000, .50)$ and $(-1,000)$ rather than as a choice between $(2,000, .50)$ and $(1,000)$. As we have seen, the former representation induces more adventurous choices than the latter.

A change of reference point alters the preference order for prospects. In particular, the present theory implies that a negative translation of a choice 
problem, such as arises from incomplete adaptation to recent losses, increases risk seeking in some situations. Specifically, if a risky prospect $(x, p ;-y, 1-p)$ is just acceptable, then $(x-z, p ;-y-z, 1-p)$ is preferred over $(-z)$ for $x, y, z>$ 0 , with $x>z$.

To prove this proposition, note that

$$
V(x, p ; y, 1-p)=0 \quad \text { iff } \quad \pi(p) v(x)=-\pi(1-p) v(-y) .
$$

Furthermore,

$$
\begin{aligned}
V(x- & z, p ;-y-z, 1-p) \\
= & \pi(p) v(x-z)+\pi(1-p) v(-y-z) \\
> & \pi(p) v(x)-\pi(p) v(z)+\pi(1-p) v(-y) \\
& +\pi(1-p) v(-z) \quad \text { by the properties of } v, \\
= & -\pi(1-p) v(-y)-\pi(p) v(z)+\pi(1-p) v(-y) \\
& +\pi(1-p) v(-z) \quad \text { by substitution, } \\
= & -\pi(p) v(z)+\pi(1-p) v(-z) \\
> & v(-z)[\pi(p)+\pi(1-p)] \quad \text { since } v(-z)<-v(z), \\
> & v(-z) \quad \text { by subcertainty. }
\end{aligned}
$$

This analysis suggests that a person who has not made peace with his losses is likely to accept gambles that would be unacceptable to him otherwise. The well known observation [31] that the tendency to bet on long shots increases in the course of the betting day provides some support for the hypothesis that a failure to adapt to losses or to attain an expected gain induces risk seeking. For another example, consider an individual who expects to purchase insurance, perhaps because he has owned it in the past or because his friends do. This individual may code the decision to pay a premium $y$ to protect against a loss $x$ as a choice between $(-x+y, p ; y, 1-p)$ and $(0)$ rather than as a choice between $(-x, p)$ and $(-y)$. The preceding argument entails that insurance is likely to be more attractive in the former representation than in the latter.

Another important case of a shift of reference point arises when a person formulates his decision problem in terms of final assets, as advocated in decision analysis, rather than in terms of gains and losses, as people usually do. In this case, the reference point is set to zero on the scale of wealth and the value function is likely to be concave everywhere [39]. According to the present analysis, this formulation essentially eliminates risk seeking, except for gambling with low probabilities. The explicit formulation of decision problems in terms of final assets is perhaps the most effective procedure for eliminating risk seeking in the domain of losses. 
Many economic decisions involve transactions in which one pays money in exchange for a desirable prospect. Current decision theories analyze such problems as comparisons between the status quo and an alternative state which includes the acquired prospect minus its cost. For example, the decision whether to pay 10 for the gamble $(1,000, .01)$ is treated as a choice between $(990, .01 ;-10, .99)$ and $(0)$. In this analysis, readiness to purchase the positive prospect is equated to willingness to accept the corresponding mixed prospect.

The prevalent failure to integrate riskless and risky prospects, dramatized in the isolation effect, suggests that people are unlikely to perform the operation of subtracting the cost from the outcomes in deciding whether to buy a gamble. Instead, we suggest that people usually evaluate the gamble and its cost separately, and decide to purchase the gamble if the combined value is positive. Thus, the gamble $(1,000, .01)$ will be purchased for a price of 10 if $\pi$ $(.01) v(1,000)+v(-10)>0$.

If this hypothesis is correct, the decision to pay 10 for $(1,000, .01)$, for example, is no longer equivalent to the decision to accept the gamble $(990, .01 ;-10, .99)$. Furthermore, prospect theory implies that if one is indifferent between $(x(1-$ $p), p ;-p x, 1-p)$ and $(0)$ then one will not pay $p x$ to purchase the prospect $(x, p)$. Thus, people are expected to exhibit more risk seeking in deciding whether to accept a fair gamble than in deciding whether to purchase a gamble for a fair price. The location of the reference point, and the manner in which 'choice problems are coded and edited emerge as critical factors in the analysis of decisions.

\section{Extensions}

In order to encompass a wider range of decision problems, prospect theory should be extended in several directions. Some generalizations are immediate; others require further development. The extension of equations (1) and (2) to prospects with any number of outcomes is straightforward. When the number of outcomes is large, however, additional editing operations may be invoked to simplify evaluation. The manner in which complex options, e.g., compound prospects, are reduced to simpler ones is yet to be investigated.

Although the present paper has been concerned mainly with monetary outcomes, the theory is readily applicable to choices involving other attributes, e.g., quality of life or the number of lives that could be lost or saved as a consequence of a policy decision. The main properties of the proposed value function for money should apply to other attributes as well. In particular, we expect outcomes to be coded as gains or losses relative to a neutral reference point, and losses to loom larger than gains.

The theory can also be extended to the typical situation of choice, where the probabilities of outcomes are not explicitly given. In such situations, decision weights must be attached to particular events rather than to stated probabilities, but they are expected to exhibit the essential properties that were ascribed to the weighting function. For example, if $A$ and $B$ are complementary events and neither is certain, $\pi(A)+\pi(B)$ should be less than unity-a natural analogue to subcertainty. 
The decision weight associated with an event will depend primarily on the perceived likelihood of that event, which could be subject to major biases [45]. In addition, decision weights may be affected by other considerations, such as ambiguity or vagueness. Indeed, the work of Ellsberg [10] and Fellner [12] implies that vagueness reduces decision weights. Consequently, subcertainty should be more pronounced for vague than for clear probabilities.

The present analysis of preference between risky options has developed two themes. The first theme concerns editing operations that determine how prospects are perceived. The second theme involves the judgmental principles that govern the evaluation of gains and losses and the weighting of uncertain outcomes. Although both themes should be developed further, they appear to provide a useful framework for the descriptive analysis of choice under risk.

\section{The University of British Columbia and \\ Stanford University}

Manuscript received November, 1977; final revision received March, 1978.

\section{APPENDIX $^{2}$}

In this appendix we sketch an axiomatic analysis of prospect theory. Since a complete self-contained treatment is long and tedious, we merely outline the essential steps and exhibit the key ordinal properties needed to establish the bilinear representation of equation (1). Similar methods could be extended to axiomatize equation (2).

Consider the set of all regular prospects of the form $(x, p ; y, q)$ with $p+q<1$. The extension to regular prospects with $p+q=1$ is straightforward. Let $\geqslant$ denote the relation of preference between prospects that is assumed to be connected, symmetric and transitive, and let $\simeq$ denote the associated relation of indifference. Naturally, $(x, p ; y, q) \simeq(y, q ; x, p)$. We also assume, as is implicit in our notation, that $(x, p ; 0, q) \simeq(x, p ; 0, r)$, and $(x, p ; y, 0) \simeq(x, p ; z, 0)$. That is, the null outcome and the impossible event have the property of a multiplicative zero.

Note that the desired representation (equation (1)) is additive in the probability-outcome pairs. Hence, the theory of additive conjoint measurement can be applied to obtain a scale $V$ which preserves the preference order, and interval scales $f$ and $g$ in two arguments such that

$$
V(x, p ; y, q)=f(x, p)+g(y, q) .
$$

The key axioms used to derive this representation are:

Independence: $(x, p ; y, q) \geqslant\left(x, p ; y^{\prime} q^{\prime}\right)$ iff $\left(x^{\prime}, p^{\prime} ; y, q\right) \geqslant\left(x^{\prime}, p^{\prime} ; y^{\prime}, q^{\prime}\right)$.

Cancellation: If $\left(x, p ; y^{\prime} q^{\prime}\right) \geqslant\left(x^{\prime}, p^{\prime} ; y, q\right)$ and $\left(x^{\prime}, p^{\prime} ; y^{\prime \prime}, q^{\prime \prime}\right) \geq\left(x^{\prime \prime}, p^{\prime \prime} ; y^{\prime}, q^{\prime}\right)$, then $\left(x, p ; y^{\prime \prime}, q^{\prime \prime}\right) \geq$ $\left(x^{\prime \prime}, p^{\prime \prime} ; y, q\right)$.

Solvability: If $(x, p ; y, q) \geq(z, r) \geq\left(x, p ; y^{\prime} q^{\prime}\right)$ for some outcome $z$ and probability $r$, then there exist $y^{\prime \prime}, q^{\prime \prime}$ such that

$$
\left(x, p ; y^{\prime \prime} q^{\prime \prime}\right) \simeq(z, r) .
$$

It has been shown that these conditions are sufficient to construct the desired additive representation, provided the preference order is Archimedean $[\mathbf{8 , 2 5}$. Furthermore, since $(x, p ; y, q) \simeq$ $(y, q ; x, p), f(x, p)+g(y, q)=f(y, q)+g(x, p)$, and letting $q=0$ yields $f=g$.

Next, consider the set of all prospects of the form $(x, p)$ with a single non-zero outcome. In this case, the bilinear model reduces to $V(x, p)=\pi(p) v(x)$. This is the multiplicative model, investigated in [35] and [25]. To construct the multiplicative representation we assume that the ordering of the probability-outcome pairs satisfies independence, cancellation, solvability, and the Archimedean axiom. In addition, we assume sign dependence [25] to ensure the proper multiplication of signs. It should be noted that the solvability axiom used in [35] and [25] must be weakened because the probability factor permits only bounded solvability.

${ }^{2}$ We are indebted to David H. Krantz for his help in the formulation of this section. 
Combining the additive and the multiplicative representations yields

$$
V(x, p ; y, q)=f[\pi(p) v(x)]+f[\pi(q) v(y)] .
$$

Finally, we impose a new distributivity axiom:

$$
(x, p ; y, p) \simeq(z, p) \quad \text { iff } \quad(x, q ; y, q) \simeq(z, q) .
$$

Applying this axiom to the above representation, we obtain

$$
f[\pi(p) v(x)]+f[\pi(p) v(y)]=f[\pi(p) v(z)]
$$

implies

$$
f[\pi(q) v(x)]+f[\pi(q) v(y)]=f[\pi(q) v(z)] .
$$

Assuming, with no loss of generality, that $\pi(q)<\pi(p)$, and letting $\alpha=\pi(p) v(x), \beta=\pi(p) v(y)$, $\gamma=\pi(p) v(z)$, and $\theta=\pi(q) / \pi(p)$, yields $f(\alpha)+f(\beta)=f(\gamma)$ implies $f(\theta \alpha)+f(\theta \beta)=f(\theta \gamma)$ for all $0<\theta<1$.

Because $f$ is strictly monotonic we can set $\gamma=f^{-1}[f(\alpha)+f(\beta)]$. Hence, $\theta \gamma=\theta f^{-1}[f(\alpha)+f(\beta)]=$ $f^{-1}[f(\theta \alpha)+f(\theta \beta)]$.

The solution to this functional equation is $f(\alpha)=k \alpha^{c}[1]$. Hence, $V(x, p ; y, q)=$ $k[\pi(p) v(x)]^{c}+k[\pi(q) v(y)]^{c}$, for some $k, c>0$. The desired bilinear form is obtained by redefining the scales $\pi, v$, and $V$ so as to absorb the constants $k$ and $c$.

\section{REFERENCES}

[1] ACZÉL, J.: Lectures on Functional Equations and Their Applications. New York: Academic Press, 1966.

[2] Allais, M.: "Le Comportement de l'Homme Rationnel devant le Risque, Critique des Postulats et Axiomes de l'Ecole Americaine," Econometrica, 21 (1953), 503-546.

[3] Anderson, N. H., AND J. C. Shanteau: "Information Integration in Risky Decision Making," Journal of Experimental Psychology, 84 (1970), 441-451.

[4] ARrow, K. J.: Essays in the Theory of Risk-Bearing. Chicago: Markham, 1971.

[5] BARNES, J. D., AND J. E. REINMUTH: "Comparing Imputed and Actual Utility Functions in a Competitive Bidding Setting," Decision Sciences, 7 (1976), 801-812.

[6] Coombs, C. H.: "Portfolio Theory and the Measurement of Risk," in Human Judgment and Decision Processes, ed. by M. F. Kaplan and S. Schwartz. New York: Academic Press, 1975, pp. 63-85.

[7] Davidson, D., P. Suppes, AND S. Siegel: Decision-making: An Experimental Approach. Stanford: Stanford University Press, 1957.

[8] Debreu, G.: "Topological Methods in Cardinal Utility Theory," Mathematical Methods in the Social Sciences, ed. by K. J. Arrow, S. Karlin, and P. Suppes. Stanford: Stanford University Press, 1960, pp. 16-26.

[9] Edwards, W.: "Subjective Probabilities Inferred from Decisions," Psychological Review, 69 (1962), 109-135.

[10] Ellsberg, D.: "Risk, Ambiguity and the Savage Axiorns," Quarterly Journal of Economics, 75 (1961), 643-669.

[11] Fellner, W.: "Distortion of Subjective Probabilities as a Reaction to Uncertainty," Quarterly Journal of Economics, 75 (1961), 670-690.

[12] —-: Probability and Profit-A Study of Economic Behavior Along Bayesian Lines. Homewood, Illinois: Richard D. Irwin, 1965.

[13] Fishburn, P. C.: "Mean-Risk Analysis with Risk Associated with Below-Target Returns," American Economic Review, 67 (1977), 116-126.

[14] Fishburn, P. C., AND G. A. Kochenberger: "Two-Piece von Neumann-Morgenstern Utility Functions," forthcoming.

[15] Friedman, M., AND L. J. SAVAGE: "The Utility Analysis of Choices Involving Risks," Journal of Political Economy, 56 (1948), 279-304.

[16] FuCHS, V. R.: "From Bismark to Woodcock: The "Irrational" Pursuit of National Health Insurance," Journal of Law and Economics, 19 (1976), 347-359.

[17] Galanter, E., AND P. Pliner: "Cross-Modality Matching of Money Against Other Continua," in Sensation and Measurement, ed. by H. R. Moskowitz et al. Dordrecht, Holland: Reidel, 1974, pp. 65-76. 
[18] Grayson, C. J.: Decisions under Uncertainty: Drilling Decisions by Oil and Gas Operators. Cambridge, Massachusetts: Graduate School of Business, Harvard University, 1960.

[19] Green, P. E.: "Risk Attitudes and Chemical Investment Decisions," Chemical Engineering Progress, 59 (1963), 35-40.

[20] Grether, D. M., AND C. R. Plott: "Economic Theory of Choice and the Preference Reversal Phenomenon," American Economic Review, forthcoming.

[21] Halter, A. N., AND G. W. DeAn: Decisions under Uncertainty. Cincinnati: South Western Publishing Co., 1971.

[22] HANSSON, B.: "The Appropriateness of the Expected Utility Model," Erkenntnis, 9 (1975), 175-194.

[23] Helsun, H.: Adaptation-Level Theory. New York: Harper, 1964.

[24] Keeney, R. L., AND H. RAIFFA: Decisions with Multiple Objectives: Preferences and Value Tradeoffs. New York: Wiley, 1976.

[25] Krantz, D. H., D. R. LuCE, P. Suppes, AND A. TVersky: Foundations of Measurement. New York: Academic Press, 1971.

[26] Kunreuther, H., R. Ginsberg, L. Miller, P. Sagi, P. Slovic, B. Borkan, and N. KATZ: Disaster Insurance Protection: Public Policy Lessons. New York: Wiley, 1978.

[27] Lichtenstein, S, AND P. Slovic: "Reversal of Preference Between Bids and Choices in Gambling Decisions," Journal of Experimental Psychology, 89 (1971), 46-55.

[28] MACCRIMMON, K. R., AND S. LARSSON: "Utility Theory: Axioms versus Paradoxes," in Expected Utility Hypothesis and the Allais Paradox, ed. by M. Allais and O. Hagen, forthcoming in Theory and Decision.

[29] MARkowitz, H.: "The Utility of Wealth," Journal of Political Economy, 60 (1952), 151-158.

[30] — Portfolio Selection. New York: Wiley, 1959.

[31] MCGlothlin, W. H.: "Stability of Choices among Uncertain Alternatives," American Journal of Psychology, 69 (1956), 604-615.

[32] MOSTEller, F., AND P. NOGEe: "An Experimental Measurement of Utility," Journal of Political Economy, 59 (1951), 371-404.

[33] Pratt, J. W.: "Risk Aversion in the Small and in the Large," Econometrica, 32 (1964), 122-136.

[34] RAIfFA H.: Decision Analysis: Introductory Lectures on Choices Under Uncertainty. Reading, Massachusetts: Addison-Wesley, 1968.

[35] Roskies, R.: "A Measurement Axiomatization for an Essentially Multiplicative Representation of Two Factors," Journal of Mathematical Psychology, 2 (1965), 266-276.

[36] Savage, L. J.: The Foundations of Statistics. New York: Wiley, 1954.

[37] Slovic, P., B. FischHOFF, S. Lichtenstein, B. CorrigAn, AND B. CoOMBs: "Preference for Insuring Against Probable Small Losses: Insurance Implications," Journal of Risk and Insurance, 44 (1977), 237-258.

[38] Slovic, P., AND A. TVErsky: "Who Accepts Savage's Axiom?," Behavioral Science, 19 (1974), 368-373.

[39] SPETZler, C. S.: "The Development of Corporate Risk Policy for Capital Investment Decisions," IEEE Transactions on Systems Science and Cybernetics, SSC-4 (1968), 279-300.

[40] Swalm, R. O.: "Utility Theory-Insights into Risk Taking," Harvard Business Review, 44 (1966), 123-136.

[41] ToBIN, J.: "Liquidity Preferences as Behavior Towards Risk," Review of Economic Studies, 26 (1958), 65-86.

[42] TVersky, A.: "Additivity, Utility, and Subjective Probability," Journal of Mathematical Psychology, 4 (1967), 175-201.

[43] —_: "Intransitivity of Preferences," Psychological Review, 76 (1969), 31-48.

[44] —- "Elimination by Aspects: A Theory of Choice," Psychological Review, 79 (1972), 281-299.

[45] TVERsky, A., AND D. KAHNEMAN: "Judgment under Uncertainty: Heuristics and Biases," Science, 185 (1974), 1124-1131.

[46] VAN DAM, C.: "Another Look at Inconsistency in Financial Decision-Making," presented at the Seminar on Recent Research in Finance and Monetary Economics, Cergy-Pontoise, March, 1975.

[47] von Neumann, J., And O. Morgenstern, Theory of Games and Economic Behavior, Princeton: Princeton University Press, 1944.

[48] Williams, A. C.: "Attitudes toward Speculative Risks as an Indicator of Attitudes toward Pure Risks," Journal of Risk and Insurance, 33 (1966), 577-586. 